\title{
Environmental and economic performance of hybrid power-to-liquid and biomass-to-liquid fuel production in the United States
}

\section{Supplementary Information}

Stewart A. Isaacs ${ }^{1}$, Mark D. Staples ${ }^{1 *}$, Florian Allroggen ${ }^{1,2}$, Dharik S. Mallapragada ${ }^{3}$, Christoph P. Falter ${ }^{1}$, Steven R.H. Barrett ${ }^{1,2,4}$

${ }^{1}$ Laboratory for Aviation and the Environment, Department of Aeronautics and Astronautics, Massachusetts Institute of Technology

${ }^{2}$ Joint Program on the Science and Policy of Global Change, Massachusetts Institute of Technology

${ }^{3}$ MIT Energy Initiative, Massachusetts Institute of Technology, Cambridge, MA 02139, USA

${ }^{4}$ Department of Mechanical Engineering, Seoul National University, Seoul, South Korea

Number of pages: 32

Number of Notes: 3

Number of Figures: 5

Number of Tables: 36

\footnotetext{
*Corresponding Author, mstaples@alum.mit.edu
} 


\section{Supplementary Notes}

Supplementary Note 1 | Technology Uncertainty and Stochastic Modeling

Uncertainty is quantified in the process mass and energy balances. Each process technology in a pathway was characterized by parameters to quantify the associated energy and mass flows. Parameter values are sourced from literature to estimate performance for each technology. These values are represented stochastically using a uniform distribution between the maximum and minimum values reported in the literature for each parameter. All values used in the model are included in Supplementary Tables 5-17.

A monte-carlo simulation is done for each pathway using 1000 simulations. 1000 simulations allow the maximum change in variance within a pathway's net mass and energy flows to be less than $0.1 \%$ for all pathways.

\section{Supplementary Note 2 | Direct Air Carbon Capture Modeling}

There are currently two major technologies being pursued commercially to extract carbon from the atmosphere. The first uses a solid sorbent to capture carbon, whereas the other uses an aqueous solution. ${ }^{1-2}$ Although more energy efficient direct air capture technologies are under development, we limit our analysis to these as they have near-term commercial viability. ${ }^{3}$ Rather than selecting one of these two technologies, this analysis estimates the energy balance by adopting the theoretical framework proposed by House et al. (2011). ${ }^{4}$ This framework assumes a minimum energetic work required to extract carbon based on its concentration in the incoming gas stream. The total energy required is then calculated by varying $2^{\text {nd }}$ law efficiencies, likely between $5-9 \%$ for direct air capture (House et al., 2011). Equations 1 and 2 show how these values are used to calculate energy consumption,

$$
\begin{gathered}
E_{S p_{C O 2}=}=\frac{W_{\min }}{\eta} \\
E_{\text {total }}=\frac{m_{\mathrm{CO}_{2} E_{S p C O 2}}}{m w_{C O 2}}
\end{gathered}
$$

where $E_{s p, C O 2}$ is the specific energy per mole of $\mathrm{CO}_{2}$ captured, $W_{\min }$ is the minimum work required to extract $\mathrm{CO}_{2}$ based on its concentration in the gas stream, $\eta$ is the second law efficiency, $m_{\mathrm{CO} 2}$ is the mass of $\mathrm{CO}_{2}, \mathrm{mwCO}_{\mathrm{CO}}$ is the molecular weight of $\mathrm{CO}_{2}$ in mass per mole and $E_{\text {total }}$ is the total energy required for the process.

Using this approach while assuming an $8 \%$ second law efficiency yields an estimate of the required energy that is within $2 \%$ of the value reported by the technology demonstration in Keith et al. (2018) for the case of a low-pressure $\mathrm{CO}_{2}$ stream with a free oxygen supply. DAC cost values from Keith et al. (2018) are used to estimate the capital cost of the process. ${ }^{1}$ 
Supplementary Note 3 | Approach for estimating cost of supplying H2 round-the-clock from renewable electricity driven-electrolysis coupled with energy storage

We assess the levelized cost of continuous $\mathrm{H} 2$ supply from renewable electricity based on the integrated design and operations optimization model described in Mallapragada et al. (2020). ${ }^{5}$ For each location and cost scenario, the model estimates the cost-optimal size and hourly operation throughout the year of co-located components (PV and/or wind capacity, electrolyzer, battery storage, compressed $\mathrm{H} 2$ storage tank, compressor prior to storage) shown in Figure 1of the main text, in order to meet the specified hourly $\mathrm{H} 2$ production requirements $(2.5$ tonnes per hour or 60 tonnes per day with $+/-10 \%$ hourly variation). The model objective function, corresponding to the sum of annualized capital and operating costs of the above components, is minimized subject to adhering to following operational constraints: 1) inter-temporal constraints governing energy storage ( $\mathrm{H}_{2}$, battery) operation, 2 ) hourly limits on $\mathrm{PV}$ and/or wind resource availability, 3) for each component, hourly power flows cannot exceed installed power capacity, 4) throughput-based energy requirements for $\mathrm{H}_{2}$ compression prior to storage, 5) minimum downtime requirements for the entire plant (12 hours) and 6) annual plant availability requirements $(95 \%$, consistent with what is typical availability of natural gas based $\mathrm{H} 2$ production facilities). Further details of the model are available in Mallapragada et al. (2020). ${ }^{5}$

Spatially-resolved levelized $\mathrm{H}_{2}$ production costs used in generating Figure 7 and Figure 8 of the main text was developed based on evaluating the design and operations optimization model for 1487 locations across the U.S. These locations correspond to points on a grid developed from evenly-spaced latitude and longitudes spanning the continental U.S. land area. See Mallapragada et al. (2020) for further details. ${ }^{5}$

For each location, hourly PV capacity factor was simulated using solar resource availability from the National Solar Radiation Database (NSRDB) in conjunction with the pvlib simulation toolbox that converts the insolation data available from NSRDB into a capacity factor time series. ${ }^{6}$ The PV capacity factor is generated for a horizontal single axis tracking system with a DC-to-AC ratio of 1.3 .

Hourly wind capacity factor data for each location is simulated using wind resource data from the NREL Wind Integration National Database (WIND) toolkit, which has resource availability for 2007-2013 weather years. The wind capacity factors are generated for a Gamesa:G126/2500 turbine with a hub height consideration of $100 \mathrm{~m}$, following the approach described in Brown and Botterud (2020). ${ }^{7}$

At each of the 1487 locations evaluated, both solar and wind capacity factor data are estimated by evaluating the resource availability at the closest locations available in the NREL NSRDB and WIND toolkit respectively. For both resources, capacity factor was estimated based on 2011 weather year, which was deemed a typical weather year from the available data set.

\section{Supplementary Figures}

Supplementary Figures 1-5 | Process Flow Diagrams 
The mass and energy flows of the electrofuel production pathways are visualized in Figures S1S5.

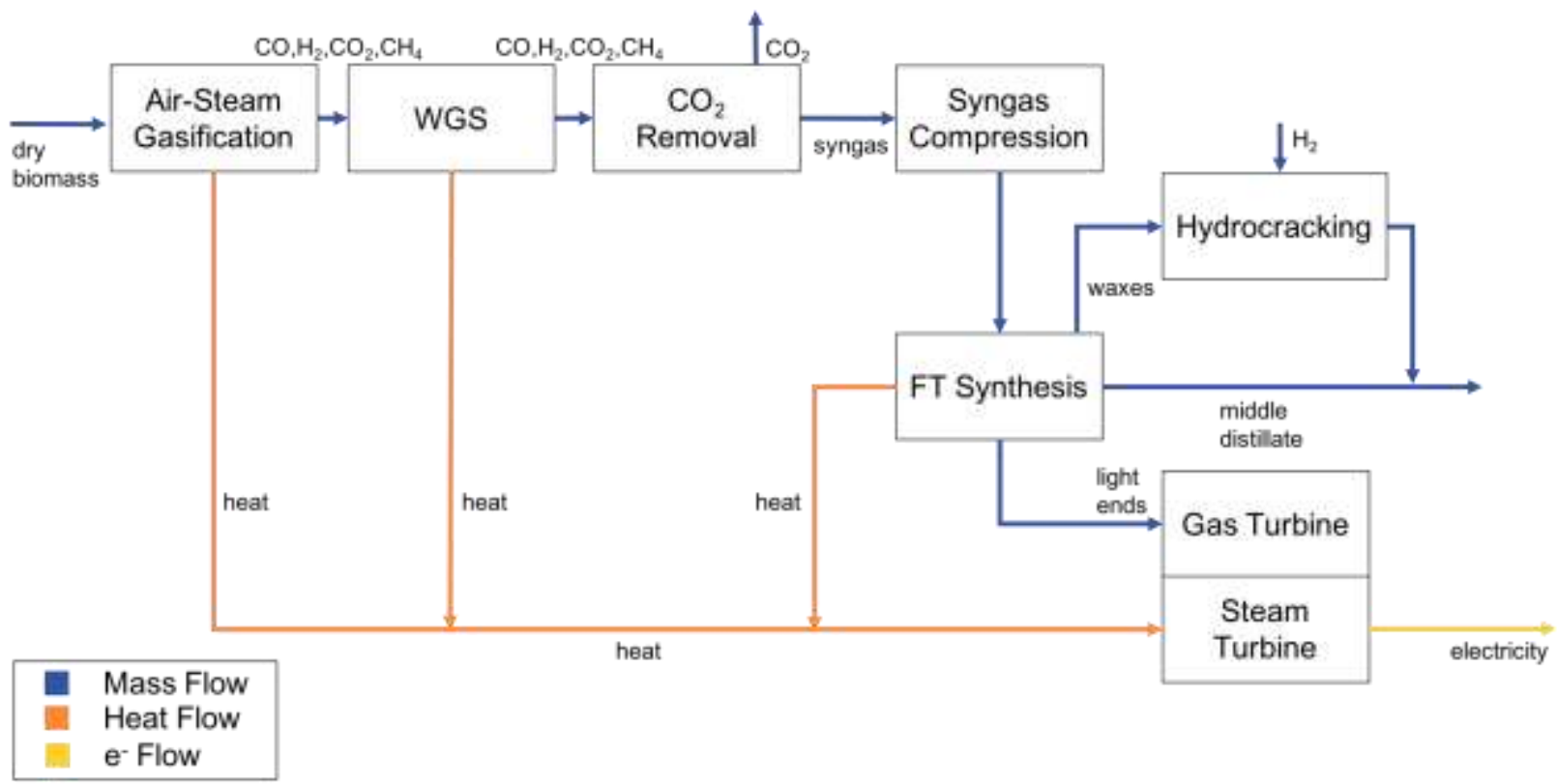

Figure S1. Biomass-to-Liquid (BtL) production pathway. Where FT Synthesis is the FT Synthesis unit, WGS is the Water Gas Shift unit, and Gasification is assumed to use an Air-Steam gasification process unit.

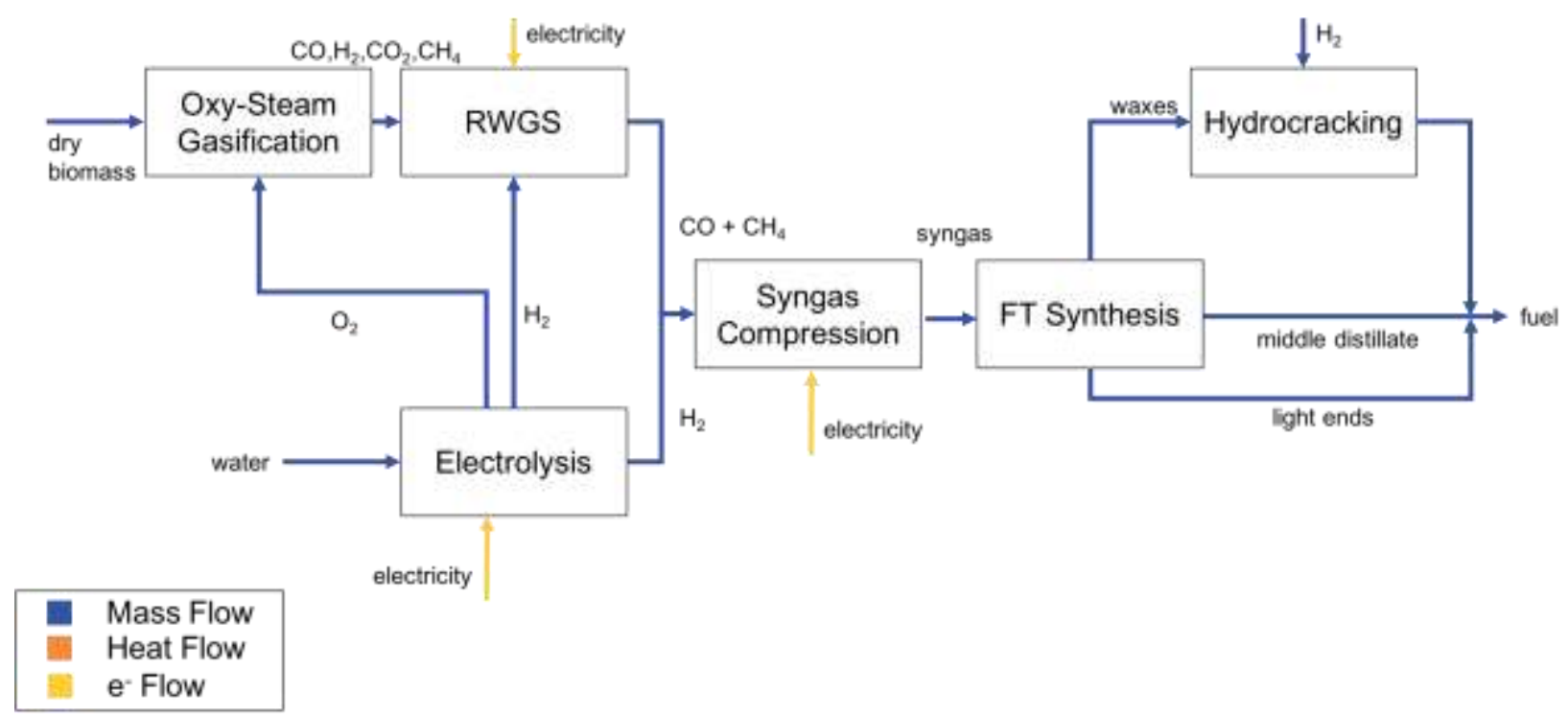

Figure S2. Power and Biomass-to-Liquid (PBtL) production pathway. Where FT Synthesis is the FT Synthesis unit, RWGS is the Reverse Water Gas Shift unit, and Gasification is assumed to use an Oxy-Steam gasification process unit. 


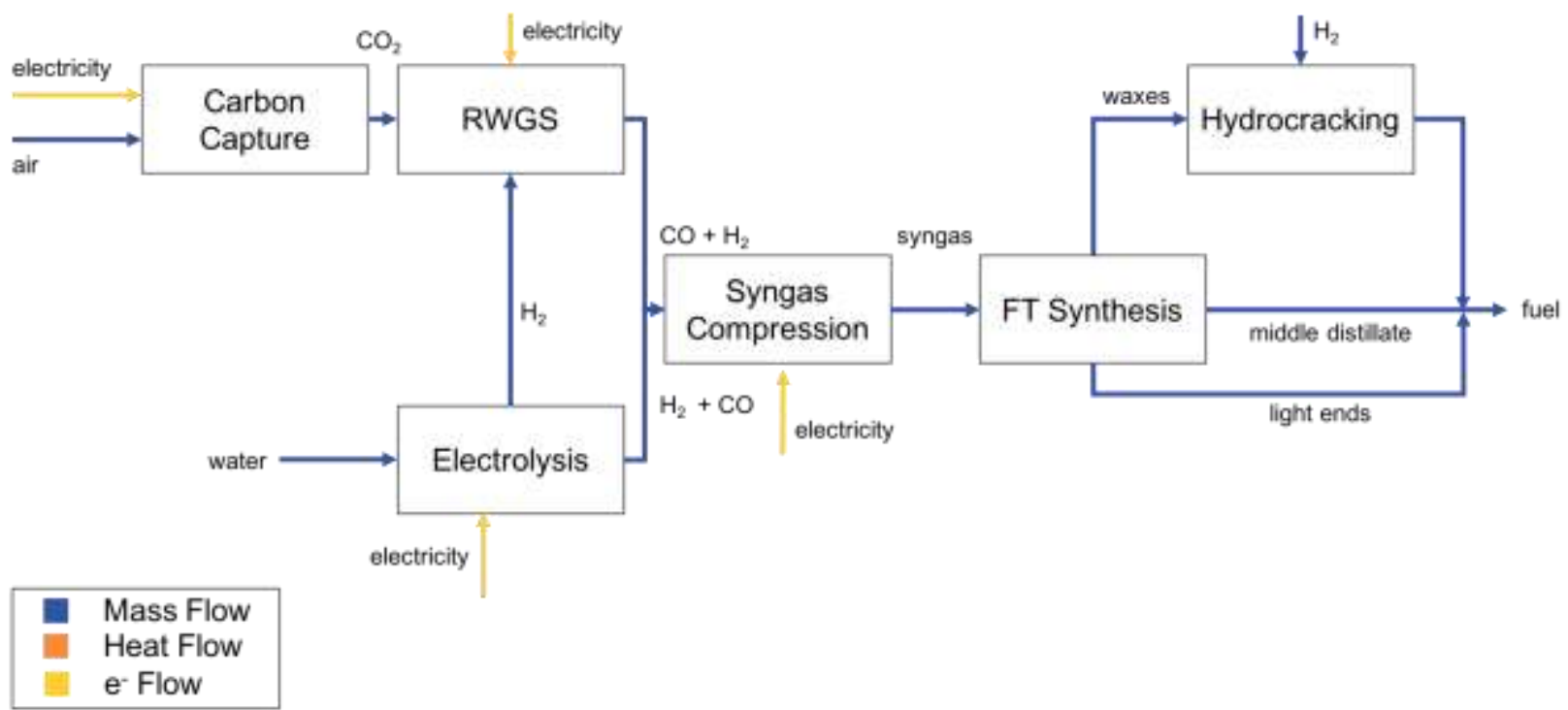

Figure S3. Two-Step Power-to-Liquid (PtL) production pathway using RWGS and electrolysis. Where FT Synthesis is the FT Synthesis unit, RWGS is the Reverse Water Gas Shift unit and the carbon capture is done with a Direct-Air Capture system.
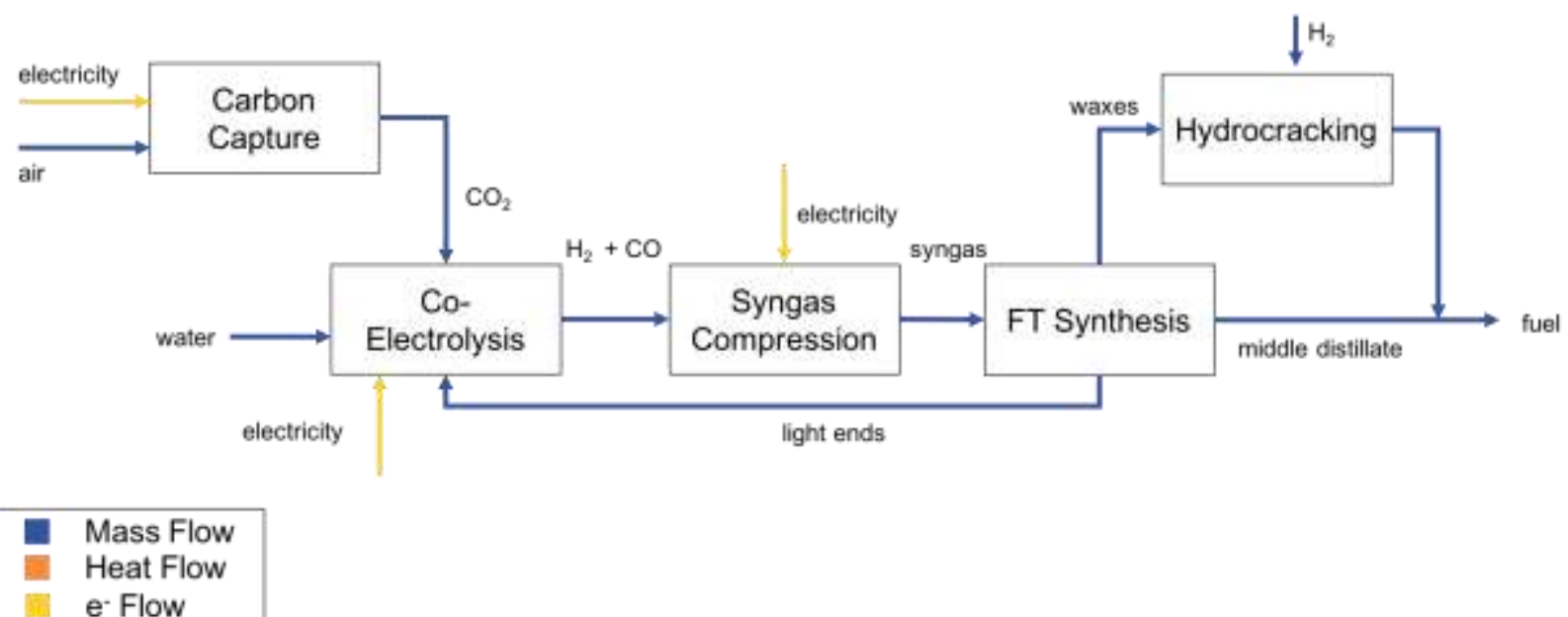

Figure S4. One-Step PtL production pathway using co-electrolysis. Where FT Synthesis is the FT Synthesis unit and the carbon capture is done with a Direct-Air Capture system. 


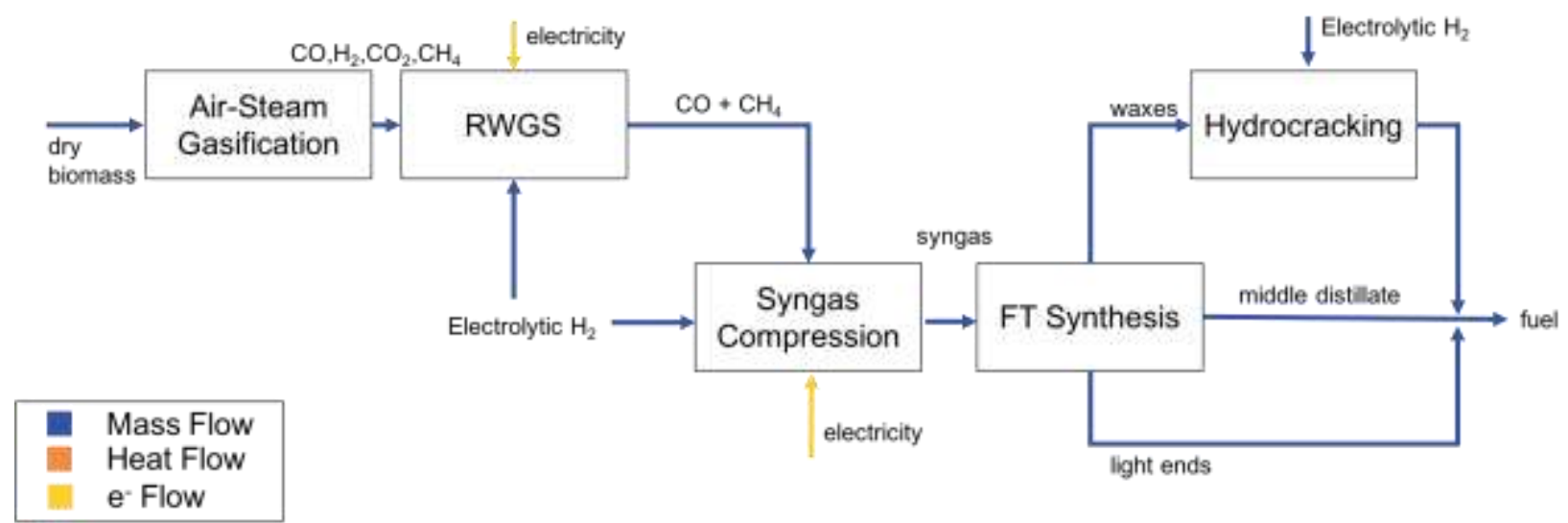

Figure S5. Low-Carbon Hydrogen PBtL pathway. Where FT Synthesis is the FT Synthesis unit, RWGS is the Reverse Water Gas Shift unit, and Gasification is assumed to use an Air-Steam gasification process unit. 


\section{Supplementary Tables}

Supplementary Table S1 | Electricity Generation Emissions Indices

\begin{tabular}{|c|c|c|}
\hline $\begin{array}{c}\text { Electricity Generation } \\
\text { Source }\end{array}$ & $\begin{array}{c}\text { Emission Intensity } \\
{\left[\mathrm{gcoze}_{\mathbf{k}} \mathbf{k W h}\right]}\end{array}$ & Source \\
\hline Coal & 980 & 9 \\
\hline Natural Gas-CT & 670 & 9 \\
\hline Natural Gas-CC & 450 & 10 \\
\hline Petroleum & 840 & 11 \\
\hline Nuclear & 12 & 12 \\
\hline Hydropower & 7 & 12 \\
\hline Biomass & 40 & 13 \\
\hline Geothermal & 22 & 14,12 \\
\hline PV-Rooftop & 44 & 16 \\
\hline PV-Utility & 26 & 17 \\
\hline CSP & 28 & 17 \\
\hline Wind-Onshore & 11 & \\
\hline Wind-Offshore & 11 & \\
\hline
\end{tabular}

Supplementary Table S2 | Levelized Cost of Electricity for Renewable Energy Generation

\begin{tabular}{|c|c|c|}
\hline Electricity Generation Source & LCOE [\$/MWh] & Source \\
\hline PV & 52.7 & 18 \\
\hline Solar Thermal & 137.5 & 18 \\
\hline Wind (onshore) & 51.3 & 18 \\
\hline Wind (offshore) & 110.4 & 18 \\
\hline Geothermal & 40.5 & 18 \\
\hline Nuclear & 73.5 & 18 \\
\hline Hydroelectric & 49.6 & \\
\hline
\end{tabular}


Supplementary Tables S3 \& S4 | Dedicated Renewable Energy Results

Biomass is assumed to be switchgrass at a cost $\$ 50$ per ton in all scenarios.

Table S3. Techno-economic assessment results when using dedicated renewable power for electrofuel production.

\begin{tabular}{|c|c|c|c|c|c|c|}
\hline Pathway & $\begin{array}{c}\text { Electricity } \\
\text { Source }\end{array}$ & $\begin{array}{c}\text { Electricity } \\
\text { Cost } \\
{[\$ / \mathrm{MWh}]}\end{array}$ & $\begin{array}{c}\text { CAPEX } \\
{[\mathrm{mm} \$]}\end{array}$ & $\begin{array}{c}\text { OPEX } \\
{[\mathrm{mm} \$ / \text { day }]}\end{array}$ & $\begin{array}{c}\text { Median } \\
\text { MSP }[\$ / \mathrm{L}]\end{array}$ & $\begin{array}{c}\text { Abatement } \\
{[\$ / \text { tCO2e] }}\end{array}$ \\
\hline BtL & Wind & 51.3 & 478 & 0.01 & 0.82 & 385 \\
\hline PBtL & Wind & 51.3 & 441 & 0.16 & 1.65 & 564 \\
\hline PtL ElecRWGS & Wind & 51.3 & 773 & 0.27 & 2.89 & 963 \\
\hline PtL CoElec & Wind & 51.3 & 973 & 0.17 & 3.07 & 1074 \\
\hline BtL & PV & 52.7 & 478 & 0.01 & 0.82 & 383 \\
\hline PtL ElecRWGS & PV & 52.7 & 441 & 0.16 & 1.67 & 617 \\
\hline PtL CoElec & PV & 52.7 & 973 & 0.18 & 3.09 & 1227 \\
\hline BtL & Geothermal & 40.5 & 478 & 0.01 & 0.85 & 399 \\
\hline PBtL & Geothermal & 40.5 & 441 & 0.13 & 1.48 & 536 \\
\hline PtL ElecRWGS & Geothermal & 40.5 & 773 & 0.21 & 2.56 & 959 \\
\hline PtL CoElec & Geothermal & 40.5 & 973 & 0.14 & 2.84 & 1087 \\
\hline BtL & Nuclear & 73.5 & 478 & 0.00 & 0.76 & 354 \\
\hline PBtL & Nuclear & 73.5 & 441 & 0.22 & 1.99 & 684 \\
\hline PtL ElecRWGS & Nuclear & 73.5 & 773 & 0.38 & 3.58 & 1202 \\
\hline
\end{tabular}


Table. S4 Lifecycle analysis results when using dedicated renewable power for electrofuel production

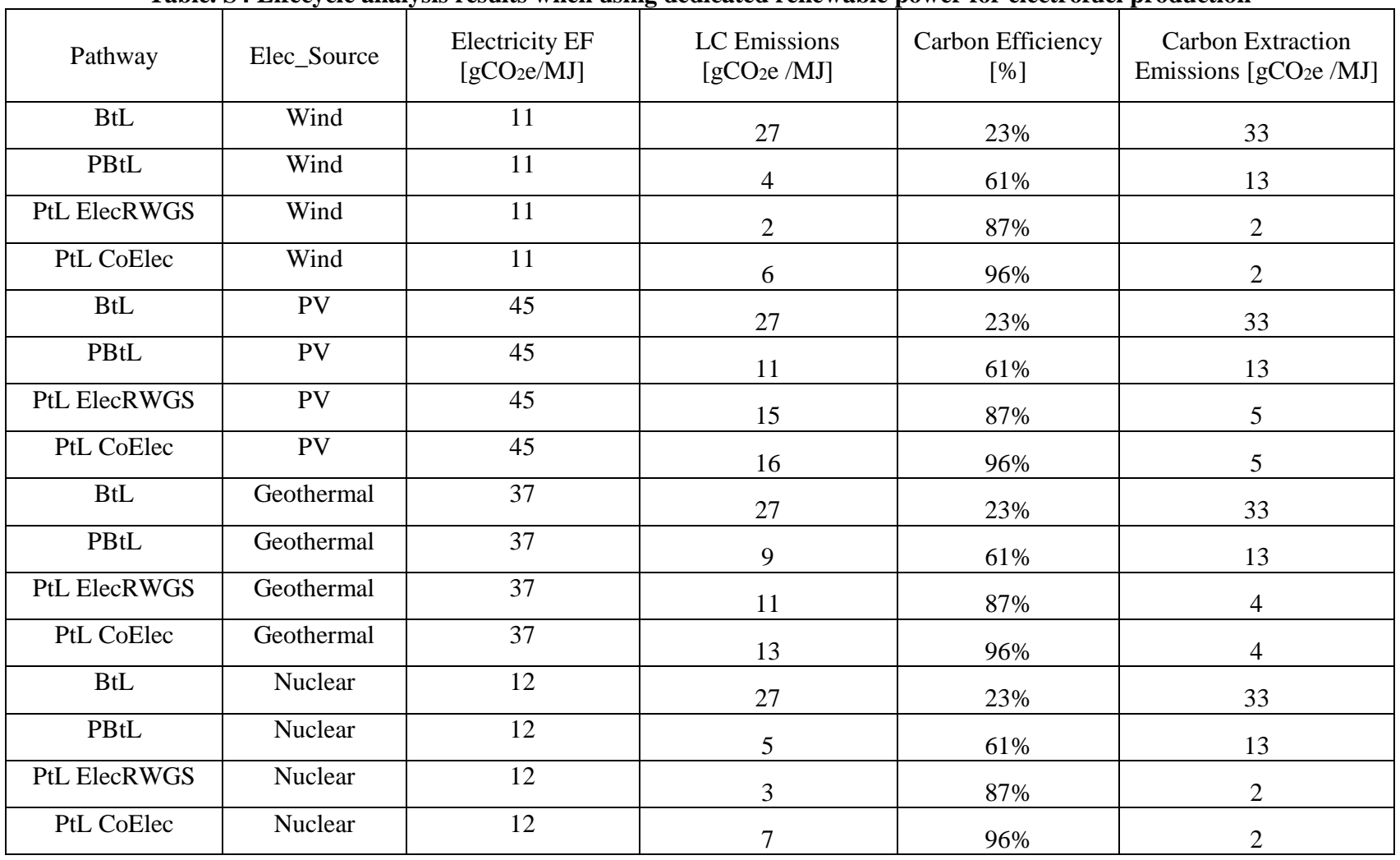

Supplementary Tables S5 - S17 | Mass and Energy Balances

Table S5. Compressor assumptions

\begin{tabular}{|c|c|c|c|c|c|c|}
\hline Variable & Min & Max & Units & Description & \multicolumn{2}{|c|}{19} \\
\hline efficiency & 70 & 85 & $\%$ & $\begin{array}{c}\text { Isentropic Efficiency for } \\
\text { compressor }\end{array}$ \\
\hline $\mathrm{Z}$ & 0.9 & 1.1 & $\mathrm{~N} / \mathrm{A}$ & Compression Factor & & 20 \\
\hline $\mathrm{n}$ & 1.05 & 360 & $\mathrm{~N} / \mathrm{A}$ & Heat Capacity Ratio & 20 & 2 \\
\hline $\begin{array}{c}\text { Specific Energy } \\
\mathrm{CO}_{2}\end{array}$ & $\mathrm{~kJ} / \mathrm{kg}$ & $\mathrm{CO}_{2} \mathrm{CCS}$ Compression Energy & 20 \\
\hline
\end{tabular}


Table S6. Air-Steam Gasification process assumptions

\begin{tabular}{|c|c|c|c|c|c|}
\hline Variable & Min & Max & Units & Description & Source \\
\hline Gas Yield & 2.23 & 2.34 & $\begin{array}{c}\mathrm{Nm}^{3} \\
\text { Syngas/kg } \\
\text { Biomass } \\
\end{array}$ & & 21 \\
\hline Steam Ratio & 1.35 & 2.7 & $\mathrm{~kg} / \mathrm{kg}$ & $\begin{array}{c}\text { Ratio of mass of steam required per } \\
\mathrm{kg} \text { Biomass in reaction }\end{array}$ & 21 \\
\hline CGE & 52.5 & 71.5 & $\%$ & Cold gas efficiency of the process & 22 \\
\hline Tar yield & 5.7 & 12.4 & $\mathrm{~g} / \mathrm{Nm}^{3}$ & $\begin{array}{c}\text { Tar yield per } \mathrm{Nm}^{\wedge} 3 \text { of syngas } \\
\text { produced }\end{array}$ & 22 \\
\hline Char yield & & & $\mathrm{kg} / \mathrm{kg}$ & & \\
\hline \multicolumn{6}{|c|}{ Syngas Volume $\%$} \\
\hline $\mathrm{H}_{2}$ & 52.4 & & $\begin{array}{l}\text { \% element / } \\
\mathrm{Nm}^{3} \text { Syngas }\end{array}$ & $\begin{array}{l}\text { volume fraction of the element in } \\
\text { the overall syngas mixture }\end{array}$ & 23 \\
\hline $\mathrm{CO}$ & 28.7 & & $\begin{array}{l}\% \text { element / } \\
\mathrm{Nm}^{3} \text { Syngas }\end{array}$ & $\begin{array}{l}\text { volume fraction of the element in } \\
\text { the overall syngas mixture }\end{array}$ & 23 \\
\hline $\mathrm{CO}_{2}$ & 16.8 & & $\begin{array}{l}\% \text { element / } \\
\mathrm{Nm}^{3} \text { Syngas }\end{array}$ & $\begin{array}{l}\text { volume fraction of the element in } \\
\text { the overall syngas mixture }\end{array}$ & 23 \\
\hline $\mathrm{CH}_{4}$ & 2.1 & & $\begin{array}{l}\% \text { element / } \\
\mathrm{Nm}^{3} \text { Syngas }\end{array}$ & $\begin{array}{l}\text { volume fraction of the element in } \\
\text { the overall syngas mixture }\end{array}$ & 23 \\
\hline
\end{tabular}

Table S7. Oxy-Steam Gasification process assumptions

\begin{tabular}{|c|c|c|c|c|c|}
\hline Variable & Min & Max & Units & Description & Source \\
\hline Gas Yield & 1.24 & 1.62 & $\begin{array}{c}\mathrm{Nm}^{3} \\
\text { Syngas } / \mathrm{kg} \\
\text { Biomass } \\
\end{array}$ & & 22 \\
\hline Steam Ratio & 1.04 & 1.42 & $\mathrm{~kg} / \mathrm{kg}$ & $\begin{array}{l}\text { Ratio of mass of steam } \\
\text { required per kg Biomass in } \\
\text { reaction }\end{array}$ & 22 \\
\hline Oxy Ratio & 0.2 & 0.5 & $\mathrm{~kg} / \mathrm{kg}$ & $\begin{array}{l}\text { Ratio of mass of oxygen } \\
\text { required per kg of Biomass }\end{array}$ & 22 \\
\hline CGE & 52.5 & 71.5 & $\%$ & $\begin{array}{l}\text { Cold gas efficiency of the } \\
\text { process }\end{array}$ & 22 \\
\hline Tar yield & 5.7 & 12.4 & $\mathrm{~g} / \mathrm{Nm}^{3}$ & $\begin{array}{l}\text { Tar yield per } \mathrm{Nm}^{3} \text { of syngas } \\
\text { produced }\end{array}$ & 22 \\
\hline Char yield & & & $\mathrm{kg} / \mathrm{kg}$ & & \\
\hline \multicolumn{6}{|c|}{ Syngas Volume $\%$} \\
\hline $\mathrm{H} 2$ & 31 & & $\begin{array}{l}\% \text { element / } \\
\mathrm{Nm}^{3} \text { Syngas }\end{array}$ & $\begin{array}{l}\text { volume fraction of the element } \\
\text { in the overall syngas mixture }\end{array}$ & 23 \\
\hline $\mathrm{CO}$ & 38.6 & & $\begin{array}{l}\% \text { element / } \\
\mathrm{Nm}^{3} \text { Syngas }\end{array}$ & $\begin{array}{l}\text { volume fraction of the element } \\
\text { in the overall syngas mixture }\end{array}$ & 23 \\
\hline $\mathrm{CO} 2$ & 27.2 & & $\begin{array}{l}\% \text { element / } \\
\mathrm{Nm}^{3} \text { Syngas }\end{array}$ & $\begin{array}{l}\text { volume fraction of the element } \\
\text { in the overall syngas mixture }\end{array}$ & 23 \\
\hline $\mathrm{CH} 4$ & 3.1 & & $\begin{array}{l}\% \text { element / } \\
\mathrm{Nm}^{3} \text { Syngas }\end{array}$ & $\begin{array}{l}\text { volume fraction of the element } \\
\text { in the overall syngas mixture }\end{array}$ & 23 \\
\hline
\end{tabular}


Table S8. Water Gas Shift reaction assumptions.

\begin{tabular}{|c|c|c|c|c|}
\hline Variable & Value & Units & Description & Source \\
\hline Energy WGS & -47.4 & $\mathrm{KJ} / \mathrm{mol}$ & $\begin{array}{l}\text { Molar specific energy of } \\
\text { reaction }\end{array}$ & 24 \\
\hline $\mathrm{H}_{2}$ ratio & 2 & $\begin{array}{c}\% \mathrm{~mol} \mathrm{H}_{2} / \% \mathrm{~mol} \\
\mathrm{CO}\end{array}$ & Molar ratio of $\mathrm{H}_{2}$ to $\mathrm{CO}$ & \\
\hline Catalyst Type & $\begin{array}{c}\mathrm{Cu}-\mathrm{ZnO}-\mathrm{Al} 2 \mathrm{O} 3 \\
\text { (EX-2248)Sud } \\
\text { Chemie }\end{array}$ & N/A & $\begin{array}{l}\text { The catalyst referenced in } \\
\text { Smith et al }\end{array}$ & 24 \\
\hline
\end{tabular}

Table S9. Reverse Water Gas Shift reactor assumptions

\begin{tabular}{|c|c|c|c|c|c|c|}
\hline Variable & Min & Max & Units & Description & Source 1 & Source 2 \\
\hline $\begin{array}{l}\text { Energy } \\
\text { RWGS }\end{array}$ & 41 & & $\mathrm{KJ} / \mathrm{mol}$ & $\begin{array}{c}\text { Molar specific energy of } \\
\text { reaction }\end{array}$ & $\begin{array}{c}\text { Gold and } \\
\text { Nickel } \\
\text { Catalysts: } \\
\text { Tables 5-2 and } \\
5-3\end{array}$ \\
\hline $\mathrm{T}$ & 1023.15 & 1173.15 & $\mathrm{~K}$ & Temperature of Reaction & 26 & 24 \\
\hline $\mathrm{X} \mathrm{CO}_{2}$ & 28 & 55 & $\%$ & Conversion Rate of Reaction & 26 & $\begin{array}{c}\text { Looked at CO } \\
\text { selectivities of } \\
100\end{array}$ \\
\hline
\end{tabular}

Table S10. Steam-Methane Reformation process assumptions. These values are used to calculate the heat energy consumed when reforming Fischer-Tropsch gases (also known as light ends) in a solid oxided electrolysis unit

\begin{tabular}{|c|c|c|c|c|c|}
\hline Variable & Min & Max & Units & Description & Source \\
\hline $\mathrm{T}$ & 1023 & 1073 & $\mathrm{~K}$ & Temperature of reaction & 27 \\
\hline Energy of reaction & 206 & & $\mathrm{~kJ} / \mathrm{mol}$ & Molar specific energy of reaction & 28 \\
\hline $\begin{array}{c}\text { Efficiency of } \\
\text { reaction }\end{array}$ & 65 & 75 & $\%$ & Overall efficiency of reaction & 28 \\
\hline
\end{tabular}

Table S11. Gas Turbine

\begin{tabular}{|c|c|c|c|c|c|}
\hline Variable & Min & Max & Units & Description & Source \\
\hline Turbine efficiency & 29 & 43 & $\%$ & $\begin{array}{c}\text { Overall Electrical } \\
\text { Energy out over the } \\
\text { LHV of fuel in }\end{array}$ & 20,29 \\
\hline
\end{tabular}

Table S12. Heat Recovery Steam Turbine

\begin{tabular}{|c|c|c|c|c|c|}
\hline Variable & Min & Max & Units & Description & Source \\
\hline Turbine efficiency & 29 & 34 & $\%$ & $\begin{array}{c}\text { Electrical Energy } \\
\text { out over the heat } \\
\text { energy in }\end{array}$ & 20 \\
\hline
\end{tabular}


Table S13. PEM Electrolysis

\begin{tabular}{|c|c|c|c|c|c|c|}
\hline Variable & Min & $\operatorname{Max}$ & Units & Description & Source & Source 2 \\
\hline $\mathrm{H}_{2}$ ratio & 2 & & $\% \mathrm{molH}_{2} / \% \mathrm{molCO}$ & Molar ratio of $\mathrm{H}_{2}$ to $\mathrm{CO}$ & & \\
\hline $\mathrm{T}$ & 1073 & 1273 & $\mathrm{~K}$ & & 30 & \\
\hline delta $S$ & 0.163 & & $\mathrm{~kJ} / \mathrm{mol}-\mathrm{K}$ & $\begin{array}{c}\text { Change in Entropy of } \\
\text { reaction }\end{array}$ & & \\
\hline $\begin{array}{l}\text { Energy of } \\
\text { Reaction }\end{array}$ & 242 & & $\mathrm{~kJ} / \mathrm{mol}$ & $\begin{array}{c}\text { Total Molar specific } \\
\text { energy of reaction } \\
\text { (LHV) }\end{array}$ & & \\
\hline $\mathrm{O}_{2}$ to $\mathrm{H}_{2}$ ratio & 0.5 & & $\mathrm{~mol} / \mathrm{mol}$ & $\begin{array}{l}\text { Molar ratio of Oxygen to } \\
\text { Hydrogen in reaction }\end{array}$ & 31 & \\
\hline Specific energy & 4.35 & 4.70 & $\mathrm{kwh} / \mathrm{Nm}^{3}$ & $\begin{array}{l}\text { Specific Energy of } \mathrm{H} 2 \\
\text { production }\end{array}$ & 32 & \\
\hline $\begin{array}{l}\text { Specific energy } \\
\text { (2050) }\end{array}$ & 4.00 & 4.42 & $\mathrm{kwh} / \mathrm{Nm}^{3}$ & $\begin{array}{l}\text { Specific Energy of } \mathrm{H} 2 \\
\text { production }\end{array}$ & 32 & \\
\hline
\end{tabular}

Table S14. Co-Electrolysis

\begin{tabular}{|c|c|c|c|c|}
\hline & & & & \\
\hline Variable & Value & Units & Description & Source \\
\hline \multicolumn{5}{|c|}{ Inputs } \\
\hline $\begin{array}{l}\mathrm{CO}_{2} \text { to Water } \\
\text { ratio }\end{array}$ & 0.865 & $\mathrm{kgH}_{2} \mathrm{O} / \mathrm{kgCO}_{2}$ & Mass ratio of INPUT $\mathrm{CO}_{2}$ to Water & 33 \\
\hline Heat Energy & 764 & $\mathrm{KJ} / \mathrm{kg}$ & $\begin{array}{c}\text { Heat input to process } 1 \mathrm{~kg} \text { of } \mathrm{CO}_{2} \\
\text { equivalent inputs }\end{array}$ & 33 \\
\hline $\begin{array}{l}\text { Electrical } \\
\text { Energy }\end{array}$ & 17355 & $\mathrm{KJ} / \mathrm{kg}$ & $\begin{array}{c}\text { Electric Input to process } 1 \mathrm{~kg} \text { of } \mathrm{CO}_{2} \\
\text { equivalent inputs }\end{array}$ & 33 \\
\hline \multicolumn{5}{|c|}{ Outputs } \\
\hline CO output & 0.6365 & $\mathrm{kgCO} / \mathrm{kg} \mathrm{CO}_{2}$ & Output $\mathrm{CO}$ per $\mathrm{kg}$ of $\mathrm{CO} 2$ & 33 \\
\hline $\mathrm{H}_{2}$ output & 0.0969 & $\mathrm{kgH} 2 / \mathrm{kg} \mathrm{CO} 2$ & Output $\mathrm{H}_{2}$ per $\mathrm{kg}$ of $\mathrm{CO} 2$ & 33 \\
\hline $\mathrm{O}_{2}$ output & 1.132 & $\mathrm{kgO} 2 / \mathrm{k} \mathrm{CO}_{2}$ & Output $\mathrm{O}_{2}$ per kg of $\mathrm{CO} 2$ & 33 \\
\hline
\end{tabular}

Table S15. Fischer-Tropsch Synthesis

\begin{tabular}{|c|c|c|c|c|c|}
\hline Variable & Min & Max & Units & Description & Source \\
\hline alpha & 0.8 & 0.9 & $\mathrm{NaN}$ & Typical Cobalt-Catalyst LTFT & 34 \\
\hline $\begin{array}{c}\text { Specific } \\
\text { Energy FT }\end{array}$ & -11195 & & $\mathrm{KJ} / \mathrm{kg}$ & $\begin{array}{l}\text { Mass specific Heat released } \\
\text { during reaction }\end{array}$ & 34 \\
\hline $\mathrm{H}_{2}$ ratio & 2 & & $\begin{array}{l}\% \mathrm{~mol} \mathrm{H}_{2} \\
1 \% \mathrm{~mol} \mathrm{CO}\end{array}$ & Molar ratio of $\mathrm{H}_{2}$ to $\mathrm{CO}$ & \\
\hline Max chain & 50 & & \# of Carbon & $\begin{array}{l}\text { The maximum carbon chain } \\
\text { length we care about }\end{array}$ & \\
\hline $\mathrm{H}_{2} \mathrm{O}$ ratio & 0.642 & & $\mathrm{~kg} / \mathrm{kg}$ & $\begin{array}{l}\text { The amount of water } \\
\text { produced per } \mathrm{kg} \text { of } \mathrm{CO}\end{array}$ & \\
\hline $\mathrm{T}$ & 473.15 & 523.15 & $\mathrm{~K}$ & $\begin{array}{c}\text { Normal Operating } \\
\text { Temperature }\end{array}$ & 34 \\
\hline $\mathrm{P}$ & 1 & 3 & Mpa & Normal Operating Pressure & 34 \\
\hline
\end{tabular}


Table S16. $\mathrm{CO}_{2}$ Removal

\begin{tabular}{|c|c|c|c|c|c|}
\hline Variable & Min & Max & Units & Description & Source \\
\hline $\begin{array}{c}\text { Separation } \\
\text { efficiency }\end{array}$ & 0.99 & $\%$ & $\begin{array}{c}\text { Removal Efficiency of } \\
\mathrm{CO}_{2}\end{array}$ & 4, Appendix Table $\mathrm{S} 3$ \\
\hline $\begin{array}{c}\text { Specific Energy } \\
\text { of } \mathrm{CO}_{2} \text { removal }\end{array}$ & 23 & 35 & $\mathrm{~kJ} / \mathrm{mol}$ & $\begin{array}{c}\text { Specific Energy of } \mathrm{CO}_{2} \\
\text { removal }\end{array}$ & \\
\hline
\end{tabular}

Table S17. $\mathrm{CO}_{2}$ Capture

\begin{tabular}{|c|c|c|c|c|c|c|}
\hline Variable & Min & Max & Units & Description & Source & Source 2 \\
\hline \multicolumn{7}{|c|}{ Flue Gas Seperation } \\
$\begin{array}{c}\text { Flue gas 2 } \\
\text { law } \\
\text { efficiency }\end{array}$
\end{tabular}


Supplementary Table S18 | Financial Assumptions

The following assumptions are sourced from the discounted cash flow rate of return method by Pearlson 2013 unless otherwise specified. ${ }^{34}$

Table S18. Financial Assumption for Techno-economic Assessment

\begin{tabular}{|c|c|c|c|}
\hline Variable & Value & Description & Source \\
\hline Equity & $20 \%$ & Equity & \\
\hline Loan Interest & $5.5 \%$ & Loan Interest & 35,36 \\
\hline Loan Term & 10 & Loan Term, years & \\
\hline WC & $5 \%$ & Working Capital (prop of FCI) & \\
\hline General Plant & 200 & General Plant & \\
\hline DPY & 10 & Depreciation Period (Years) & \\
\hline $\mathrm{CPY}$ & 3 & Construction Period (Years) & \\
\hline CY 1 & $8 \%$ & Prop Spent in Year -3 & \\
\hline CY 2 & $60 \%$ & Prop Spent in Year -2 & \\
\hline CY 3 & $32 \%$ & Prop Spent in Year -1 & \\
\hline WACC & $6 \%$ & $\begin{array}{c}\text { Use Weighted Average Cost of Capital as } \\
\text { Discount Rate }\end{array}$ & $32,37,38$ \\
\hline ITR & $17.3 \%$ & Income Tax Rate & 39 \\
\hline HPY & 8400 & Operating Hours per Year & \\
\hline VPY & 30 & Valuation Period (Years) & 38 \\
\hline Inflation & $2 \%$ & Inflation per yr & \\
\hline OPD & 350 & Operating days & \\
\hline DOC & $7.7 \%$ & Prop of FCI & \\
\hline Production Capacity 1 & 0.75 & $\begin{array}{l}50 \% \text { in the first } 6 \text { months and } 100 \% \text { rest of } \\
\text { yr1 }\end{array}$ & 35 \\
\hline Production Capacity 2 & 1 & $100 \%$ from year 2 onwards & \\
\hline
\end{tabular}

Supplementary Table S19 | State-Level Electricity Grid Scaling Factors created using data from the US EIA AEO 2019 and US EPA eGRID2016 Summary Tables ${ }^{17,39}$

\begin{tabular}{|c|c|c|}
\hline State & Emission Scaling Factor & Price Scaling Factor \\
\hline AK & 0.93 & 0.93 \\
\hline AL & 0.91 & 1.75 \\
\hline AR & 1.12 & 1.01 \\
\hline AZ & 0.93 & 0.79 \\
\hline CA & 0.45 & 1.48 \\
\hline CO & 1.47 & 0.96 \\
\hline
\end{tabular}




\begin{tabular}{|c|c|c|}
\hline $\mathrm{CT}$ & 0.50 & 1.68 \\
\hline $\mathrm{DC}$ & 0.48 & 1.08 \\
\hline $\mathrm{DE}$ & 0.89 & 1.14 \\
\hline FL & 1.02 & 0.96 \\
\hline GA & 1.00 & 0.93 \\
\hline $\mathrm{HI}$ & 1.53 & 2.32 \\
\hline IA & 1.00 & 0.79 \\
\hline ID & 0.19 & 0.91 \\
\hline IL & 0.81 & 0.90 \\
\hline IN & 1.82 & 0.83 \\
\hline KS & 1.20 & 1.02 \\
\hline $\mathrm{KY}$ & 1.96 & 0.82 \\
\hline LA & 0.88 & 0.73 \\
\hline MA & 0.82 & 1.25 \\
\hline $\mathrm{MD}$ & 1.02 & 1.19 \\
\hline $\mathrm{ME}$ & 0.35 & 1.60 \\
\hline MI & 1.10 & 1.08 \\
\hline $\mathrm{MN}$ & 1.02 & 0.97 \\
\hline MO & 1.69 & 0.84 \\
\hline MS & 0.94 & 0.95 \\
\hline MT & 1.25 & 0.86 \\
\hline $\mathrm{NC}$ & 0.87 & 0.88 \\
\hline ND & 1.67 & 0.82 \\
\hline $\mathrm{NE}$ & 1.29 & 1.52 \\
\hline $\mathrm{NH}$ & 0.32 & 1.30 \\
\hline $\mathrm{NJ}$ & 0.56 & 0.89 \\
\hline NM & 1.58 & 1.41 \\
\hline $\mathrm{NV}$ & 0.77 & 0.90 \\
\hline $\mathrm{NY}$ & 0.46 & 0.87 \\
\hline $\mathrm{OH}$ & 1.47 & 0.96 \\
\hline OK & 1.04 & 0.76 \\
\hline OR & 0.31 & 0.86 \\
\hline $\mathrm{PA}$ & 0.86 & 0.99 \\
\hline RI & 0.87 & 1.59 \\
\hline SC & 0.63 & 0.95 \\
\hline $\mathrm{SD}$ & 0.51 & 0.96 \\
\hline $\mathrm{TN}$ & 0.99 & 0.90 \\
\hline $\mathrm{TX}$ & 1.05 & 0.82 \\
\hline UT & 1.63 & 0.85 \\
\hline
\end{tabular}




\begin{tabular}{|c|c|c|}
\hline VA & 0.82 & 1.41 \\
\hline VT & 0.07 & 0.89 \\
\hline WA & 0.19 & 0.75 \\
\hline WI & 1.39 & 0.87 \\
\hline WV & 1.98 & 1.04 \\
\hline WY & 2.03 & 0.80 \\
\hline
\end{tabular}


Supplementary Tables S19 - S22 | Equipment and Material Costs

Table S19. Process Unit Capital Costs

\begin{tabular}{|c|c|c|c|c|c|c|c|c|}
\hline Process Name & $\begin{array}{l}\text { Equipment } \\
\text { Cost (EC) } \\
(\mathrm{m} \$ 2016)\end{array}$ & $\begin{array}{c}\mathrm{EC} \\
\text { ref } 2\end{array}$ & S ref & S ref Units & di & Description & Source 1 & Source 2 \\
\hline $\mathrm{CO}_{2}$ Capture & 409.7 & 574.2 & 2900 & $\mathrm{tCO}_{2} /$ day & 1 & & 1 & \\
\hline $\begin{array}{c}\text { Flue Gas } \\
\text { Capture }\end{array}$ & 117.29 & & 8000 & $\mathrm{tCO}_{2} /$ day & 1 & & 41 & \\
\hline Co-Electrolysis & 0.999 & 3.33 & 1 & MW & 1 & & 42 & 43 \\
\hline Compress & 0.61 & & 413 & $\mathrm{~kW}$ & 0.8 & $\begin{array}{c}\text { power } \\
\text { consumption }\end{array}$ & 44 & \\
\hline Gasifier & 100.71 & 129.6 & 78 & $\begin{array}{l}\mathrm{t} / \mathrm{h} \text { (slurry } \\
\text { input) }\end{array}$ & 0.7 & slurry input & 44 & 45 \\
\hline Electrolyzer & 0.80 & & 1 & MW & 1 & $\begin{array}{l}\text { installed } \\
\text { capacity }\end{array}$ & 44 & \\
\hline $\begin{array}{l}\text { Gas/Liquid } \\
\text { Separator }\end{array}$ & 0.11 & & 10 & $\mathrm{~m}$ & 0.79 & unit length & 44 & \\
\hline FT Synth & 22.12 & & 208 & $\mathrm{~m}^{\wedge} 3$ & 1 & reactor volume & 44 & \\
\hline Hydrocracking & 9.74 & & 1.13 & $\mathrm{~kg} / \mathrm{s}$ & 0.7 & feed mass flow & 44 & \\
\hline PSA & 7.37 & & 0.294 & $\mathrm{kmol} / \mathrm{s}$ & 0.74 & purge gas flow & 44 & \\
\hline RWGS & 3.00 & & 2556 & t/day & 0.65 & total mass flow & 44 & \\
\hline Selexol Unit & 74.41 & & 9909 & $\mathrm{kmol} / \mathrm{h}$ & 0.7 & $\mathrm{CO} 2$ in feed & 44 & \\
\hline Steam Turbine & 0.43 & & 10.5 & MW & 0.44 & Power output & 44 & \\
\hline Gas Turbine & 10.59 & & 25 & MW & 0.7 & power output & 44 & \\
\hline WGS Reactor & 3.48 & & 150 & $\mathrm{~kg} / \mathrm{s}$ & 0.67 & total gas feed & 44 & \\
\hline
\end{tabular}


Table S20. Indirect Capital Costs

\begin{tabular}{|c|c|c|c|c|}
\hline Indirect Cost items & $\mathrm{j}$ & Basis & $\begin{array}{l}\text { Typical } \\
\text { Value }\end{array}$ & Source \\
\hline \multicolumn{4}{|c|}{ Total direct plant costs (D) } & \\
\hline Equipment installation & 1 & $\mathrm{EC}$ & 0.47 & 44 \\
\hline Instrumentation and control & 2 & $\mathrm{EC}$ & 0.36 & 44 \\
\hline Piping (installed) & 3 & $\mathrm{EC}$ & 0.68 & 44 \\
\hline Electrical (installed) & 4 & $\mathrm{EC}$ & 0.11 & 45 \\
\hline Buildings including services & 5 & $\mathrm{EC}$ & 0.18 & 44 \\
\hline Yard Improvements & 6 & $\mathrm{EC}$ & 0.1 & 44 \\
\hline Service Facilities (installed) & 7 & $\mathrm{EC}$ & 0.55 & 44 \\
\hline \multicolumn{4}{|c|}{ Total Indirect plant costs (i) } & \\
\hline Engineering and supervision & 8 & $\mathrm{EC}$ & 0.33 & 44 \\
\hline Construction Expenses & 9 & $\mathrm{EC}$ & 0.42 & 44 \\
\hline Legal Expenses & 10 & $\mathrm{EC}$ & 0.04 & 44 \\
\hline \multicolumn{4}{|c|}{ As function of $(\mathrm{D}+\mathrm{I})$} & \\
\hline Contractor's Fee & 11 & $\mathrm{D}+\mathrm{I}$ & 0.05 & 44 \\
\hline Contingency & 12 & $\mathrm{D}+\mathrm{I}$ & 0.1 & 44 \\
\hline
\end{tabular}

Table S21. Raw Material Costs

\begin{tabular}{|c|c|c|c|c|c|}
\hline $\begin{array}{c}\text { Raw Materials \& } \\
\text { Byproducts }\end{array}$ & $\begin{array}{c}\text { Market Price } \\
(\$ 2016)\end{array}$ & $\begin{array}{l}\text { Market Price } 2 \\
\quad(\$ 2016)\end{array}$ & Units & Description & Source 1 \\
\hline Water & 0 & 3.52 & $\$ /$ ton & Water for electrolysis & 38 \\
\hline Selexol & 5502.57 & & $\$ / t$ & $1.05 \mathrm{E}-5 \mathrm{~kg} / \mathrm{kg}$ biomass & 44 \\
\hline Biomass & 121.8 & & $\$ / \mathrm{t}$ & & 44 \\
\hline Electricty Consumed & 131.3 & & \$/MWh & & \\
\hline Electricity Produced & 73.16 & 170.83 & \$/MWh & & \\
\hline $\mathrm{CaCO} 3$ & 40 & 340 & $\$ /$ ton $\mathrm{CaCO} 3$ & $\begin{array}{l}\text { Direct Air Capture } \\
\text { chemical. } 0.0156 \\
\mathrm{kgCaCO}_{3} / \mathrm{kgCO}_{2}\end{array}$ & 46 \\
\hline
\end{tabular}


Table S22. Indirect Operating Costs

\begin{tabular}{|c|c|c|c|c|}
\hline Investment Item & Basis & Typical Value 1 & Value 2 & Notes \\
\hline Operating Supervision & $\mathrm{OL}$ & 0.15 & & \\
\hline Maintenance Labor & FCI & 0.01 & 0.03 & \\
\hline Maintenance material & FCI & 0.01 & 0.03 & \\
\hline Operating supplies & M & 0.15 & & \\
\hline Laboratory charges & $\mathrm{OL}$ & 0.2 & & \\
\hline Insurance and taxes & FCI & 0.02 & & \\
\hline Plant Overhead Costs & TLC & 0.6 & & \\
\hline Administrative Costs & $\mathrm{PO}$ & 0.25 & & \\
\hline Distribution and Selling costs & NPC & 0.06 & & won't be considered \\
\hline Research and Development costs & NPC & 0.04 & & won't be considered \\
\hline
\end{tabular}

\section{Supplementary Tables S23 - S28 | Emission Quantification Values}

All values are sourced from GREET 2017.

Table S23. Fuel Specifications

\begin{tabular}{|c|c|c|c|c|}
\hline Variable & Value & Units & Description & Source \\
\hline BTU_to_MJ & 948 & BTU/MJ & & 20 \\
\hline Gasoline Density & 2.836 & $\mathrm{~kg} / \mathrm{gal}$ & & 20 \\
\hline Diesel Density & 3.017 & $\mathrm{~kg} / \mathrm{gal}$ & & 20 \\
\hline Gasoline LHV & 41.73 & $\mathrm{MJ} / \mathrm{kg}$ & & 20 \\
\hline Diesel LHV & 43.24 & $\mathrm{MJ} / \mathrm{kg}$ & & 20 \\
\hline Gasoline carbon ratio & 0.83 & $\begin{array}{c}\text { fraction by } \\
\text { weight }\end{array}$ & & 20 \\
\hline Diesel carbon ratio & 0.85 & $\begin{array}{c}\text { fraction by } \\
\text { weight }\end{array}$ & & \\
\hline
\end{tabular}


Table S24. Upstream Emissions

\begin{tabular}{|c|c|c|c|c|}
\hline Variable & Value & Units & Description & Source \\
\hline $\begin{array}{l}\text { Switchgrass } \\
\text { Farming }\end{array}$ & 82.11 & & & 20 \\
\hline $\begin{array}{c}\text { Switchgrass } \\
\text { Transportation }\end{array}$ & 93.87 & & & 20 \\
\hline Willow Farming & 40.07 & $\mathrm{gCO}_{2} \mathrm{e} / \mathrm{kg}$ & $\begin{array}{l}\text { GHG-100 Emissions per kg of } \\
\text { Willow }\end{array}$ & 29 \\
\hline $\begin{array}{c}\text { Willow } \\
\text { Transportation }\end{array}$ & 55.61 & $\mathrm{gCO}_{2} \mathrm{e} / \mathrm{kg}$ & $\begin{array}{c}\text { GHG-100 Emissions per kg of } \\
\text { Willow }\end{array}$ & 20 \\
\hline $\begin{array}{l}\text { Miscanthus } \\
\text { Farming }\end{array}$ & 46.27 & $\mathrm{gCO}_{2} \mathrm{e} / \mathrm{kg}$ & & 20 \\
\hline $\begin{array}{c}\text { Miscanthus } \\
\text { Transportation }\end{array}$ & 75.48 & $\mathrm{gCO}_{2} \mathrm{e} / \mathrm{kg}$ & & 20 \\
\hline Poplar Farming & 61.97 & $\mathrm{gCO}_{2} \mathrm{e} / \mathrm{kg}$ & & 20 \\
\hline $\begin{array}{c}\text { Poplar } \\
\text { Transportation }\end{array}$ & 14 & $\mathrm{gCO}_{2} \mathrm{e} / \mathrm{kg}$ & & 20 \\
\hline $\begin{array}{c}\text { Switchgrass Water } \\
\text { Consumption }\end{array}$ & 724.41 & $\mathrm{~cm}^{3} / \mathrm{kg}$ & Water Consumption during farming & 20 \\
\hline $\begin{array}{l}\text { Willow Water } \\
\text { Consumption }\end{array}$ & 66.88 & $\mathrm{~cm}^{3} / \mathrm{kg}$ & Water Consumption during farming & 20 \\
\hline $\begin{array}{c}\text { Miscanthus Water } \\
\text { Consumption }\end{array}$ & 560000 & $\mathrm{~cm}^{3} / \mathrm{kg}$ & Water Consumption during farming & 20 \\
\hline
\end{tabular}

Table S25. GREET 2017 Fuel Properties

\begin{tabular}{|c|c|c|c|c|}
\hline Fuel Property & Value & Units & Description & Source \\
\hline Gasoline LHV & 41.73869935 & $\mathrm{MJ} / \mathrm{kg}$ & $\mathrm{LHV}$ & 20 \\
\hline Diesel LHV & 43.24785345 & $\mathrm{MJ} / \mathrm{kg}$ & $\mathrm{LHV}$ & 20 \\
\hline Gasoline Density & 2.835 & $\mathrm{~kg} / \mathrm{gal}$ & & 20 \\
\hline Diesel Density & 3.167 & $\mathrm{~kg} / \mathrm{gal}$ & & 20 \\
\hline
\end{tabular}


Table S26. GREET 2017 Emissions Inventory for Fuel Combustion

\begin{tabular}{|c|c|c|c|cc|}
\hline & Diesel Avg & Units & Gasoline Avg & $\mathrm{g}$ \\
\hline $\mathrm{CO}_{2}$ Total & 3187.51 & $\mathrm{~g}$ & 2726.56 & $\mathrm{~g}$ \\
\hline $\mathrm{CO}_{2}$ & 3187.51 & $\mathrm{~g}$ & 2726.56 & $\mathrm{~g}$ \\
\hline Biogenic $\mathrm{CO}_{2}$ & 0.00 & $\mathrm{~g}$ & 0.00 & $\mathrm{~g}$ \\
\hline $\mathrm{VOC}$ & 0.55 & $\mathrm{~g}$ & 166.57 & $\mathrm{~g}$ \\
\hline $\mathrm{CO}$ & 8.63 & $\mathrm{~g}$ & 7.24 & $\mathrm{~g}$ \\
\hline $\mathrm{NO}_{\mathrm{x}}$ & 25.25 & $\mathrm{~g}$ & 1.19 & $\mathrm{~g}$ \\
\hline $\mathrm{PM}_{10}$ & 1.25 & $\mathrm{~g}$ & 1.19 & $\mathrm{~g}$ \\
\hline $\mathrm{PM}_{2.5}$ & 1.05 & $\mathrm{~g}$ & 0.05 & $\mathrm{~g}$ \\
\hline $\mathrm{SO}_{\mathrm{x}}$ & 0.02 & $\mathrm{~g}$ & 0.13 & $\mathrm{~g}$ \\
\hline $\mathrm{CH}_{4}$ & 0.07 & $\mathrm{~g}$ & 0.03 & $\mathrm{~g}$ \\
\hline $\mathrm{N}_{2} \mathrm{BO}$ & 0.03 & $\mathrm{~g}$ & 0.00 & $\mathrm{~g}$ \\
\hline $\mathrm{BC}$ & 0.00 & 0.00 & 0.00 & \\
\hline
\end{tabular}

Table S27. Fischer-Tropsch Diesel Combustion Emissions

\begin{tabular}{|c|c|c|}
\hline Emissions per kg of Fischer-Tropsch Diesel & & \\
\hline Well to Use & & FTD \\
\hline Emissions & 0.15 & $\mathrm{~kg}$ \\
\hline $\mathrm{CO}_{2}$ Total & 0.15 & $\mathrm{~kg}$ \\
\hline $\mathrm{CO}_{2}$ & $-4.97 \mathrm{E}-04$ & $\mathrm{~kg}$ \\
\hline Biogenic $\mathrm{CO}_{2}$ & 0.29 & $\mathrm{~g}$ \\
\hline $\mathrm{VOC}$ & 0.57 & $\mathrm{~g}$ \\
\hline $\mathrm{CO}$ & 1.71 & $\mathrm{~g}$ \\
\hline $\mathrm{NO}_{\mathrm{x}}$ & 0.14 & $\mathrm{~g}$ \\
\hline $\mathrm{PM}_{10}$ & 98.1 & $\mathrm{mg}$ \\
\hline $\mathrm{PM}_{2.5}$ & 1.69 & $\mathrm{~g}$ \\
\hline $\mathrm{SOx}$ & 0.68 & $\mathrm{~g}$ \\
\hline $\mathrm{CH}_{4}$ & 0.83 & $\mathrm{~g}$ \\
\hline $\mathrm{N}_{2} \mathrm{O}$ & 17.4 & $\mathrm{mg}$ \\
\hline $\mathrm{BC}$ & 12.83 & $\mathrm{mg}$ \\
\hline $\mathrm{POC}^{2}$ & & \\
\hline
\end{tabular}


Table S28. Global Warming Potential of Emissions Species using AR5 100 year time horizon

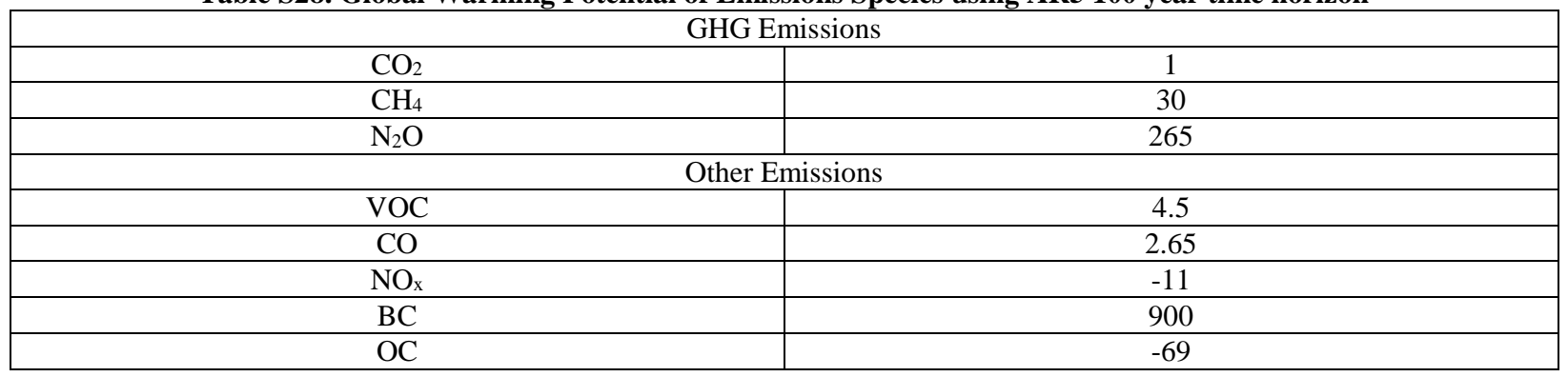

\section{Supplementary Tables S29 - S36| Low-Carbon Hydrogen Technoeconomic Assumptions}

Table S29. PEM Electrolysis Cost Assumptions - 2030

\begin{tabular}{|c|c|c|c|c|}
\hline Variable & Value & Units & Description & Source \\
\hline $\begin{array}{c}\text { Volumetric specific } \\
\text { energy }\end{array}$ & 4.70 & $\mathrm{kwh} / \mathrm{Nm}^{\wedge} 3$ & $\begin{array}{c}\text { calculated using stp and IEA } \\
\text { values }\end{array}$ & 32 \\
\hline CAPEX & 1401 & USD/kWe & hours & 32 \\
\hline Stack Lifetime & $60000-90000$ & $\%$ & $\begin{array}{c}\text { Percentage of investment } \\
\text { cost }\end{array}$ & 32 \\
\hline Stack Cost Contribution & $50 \%$ & $\%$ & $\begin{array}{c}\text { non-stack annual O\&M } \\
\text { costs as \% of CAPEX }\end{array}$ & 32 \\
\hline $\begin{array}{c}\text { Fixed Operation and } \\
\text { Maintenance Costs }\end{array}$ & $1.50 \%$ & $\%$ & $\begin{array}{c}\text { Annual O\&M costs as \% of } \\
\text { CAPE including stack } \\
\text { replacements }\end{array}$ & \\
\hline $\begin{array}{c}\text { Total Fixed Operation } \\
\text { and Maintenance Costs }\end{array}$ & $10.5 \%$ & &
\end{tabular}


Table S30. PEM Electrolysis Cost Assumptions - 2050

\begin{tabular}{|c|c|c|c|c|}
\hline Variable & Value & Units & Description & Source \\
\hline $\begin{array}{c}\text { Volumetric specific } \\
\text { energy }\end{array}$ & 4.70 & $\mathrm{kwh} / \mathrm{Nm}^{\wedge} 3$ & $\begin{array}{c}\text { calculated using stp and IEA } \\
\text { values }\end{array}$ & 32 \\
\hline CAPEX & 515 & USD/kWe & & 32 \\
\hline Stack Lifetime & $100000-150000$ & hours & & 32 \\
\hline Stack Cost Contribution & $50 \%$ & $\%$ & $\begin{array}{c}\text { Percentage of investment } \\
\text { cost }\end{array}$ & 47 \\
\hline $\begin{array}{l}\text { Fixed Operation and } \\
\text { Maintenance Costs }\end{array}$ & $1.50 \%$ & $\%$ & $\begin{array}{c}\text { non-stack annual O\&M } \\
\text { costs as \% of CAPEX }\end{array}$ & 32 \\
\hline $\begin{array}{c}\text { Total Fixed Operation } \\
\text { and Maintenance Costs }\end{array}$ & $6.3 \%$ & $\%$ & $\begin{array}{l}\text { Annual O\&M costs as } \% \text { of } \\
\text { CAPEX including stack } \\
\text { replacements }\end{array}$ & \\
\hline
\end{tabular}

Table S31. Photovoltaic Energy Generation Assumptions - 2030

\begin{tabular}{|c|c|c|c|}
\hline Variable & Value & Units & Source \\
\hline CAPEX & 800 & $\$ / \mathrm{kW} \mathrm{AC}$ & 48,49 \\
\hline $\begin{array}{c}\text { Fixed Operation } \\
\text { and Maintenance } \\
\text { Costs }\end{array}$ & 9.6 & $\begin{array}{c}\$ / \mathrm{kW}(\mathrm{AC})- \\
\mathrm{yr}\end{array}$ & 48,49 \\
\hline $\begin{array}{c}\text { Variable Operation } \\
\text { and Maintenance } \\
\text { Costs }\end{array}$ & 0 & $\$ / \mathrm{MWh}$ & 48,49 \\
\hline
\end{tabular}

Table S32. Photovoltaic Energy Generation Assumptions - 2050

\begin{tabular}{|c|c|c|c|}
\hline Variable & Value & Units & Source \\
\hline CAPEX & 600 & $\$ / \mathrm{kW} \mathrm{AC}$ & 48,49 \\
\hline $\begin{array}{c}\text { Fixed Operation } \\
\text { and Maintenance } \\
\text { Costs }\end{array}$ & 6 & $\begin{array}{c}\$ / \mathrm{kW}(\mathrm{AC})- \\
\mathrm{yr}\end{array}$ & 48,49 \\
\hline $\begin{array}{c}\text { Variable Operation } \\
\text { and Maintenance } \\
\text { Costs }\end{array}$ & 0 & $\$ / \mathrm{MWh}$ & 48,49 \\
\hline
\end{tabular}

Table S33. Wind Energy Generation Assumptions - 2030

\begin{tabular}{|c|c|c|c|}
\hline Variable & Value & Units & Source \\
\hline CAPEX & 1350 & $\$ / \mathrm{kW} \mathrm{AC}$ & 48,49 \\
\hline $\begin{array}{c}\text { Fixed Operation and } \\
\text { Maintenance Costs }\end{array}$ & 37.8 & $\begin{array}{c}\$ / \mathrm{kW}(\mathrm{AC})- \\
\mathrm{yr}\end{array}$ & 48,49 \\
\hline $\begin{array}{c}\text { Variable Operation } \\
\text { and Maintenance } \\
\text { Costs }\end{array}$ & 0 & $\$ / \mathrm{MWh}$ & 48,49 \\
\hline
\end{tabular}

Table S34. Wind Energy Generation Assumptions - 2050

\begin{tabular}{|c|c|c|c|}
\hline Variable & Value & Units & Source \\
\hline CAPEX & 1150 & $\$ / \mathrm{kW} \mathrm{AC}$ & 48,49 \\
\hline $\begin{array}{c}\text { Fixed Operation and } \\
\text { Maintenance Costs }\end{array}$ & 32.48 & $\begin{array}{c}\$ / \mathrm{kW}(\mathrm{AC})- \\
\mathrm{yr}\end{array}$ & 48,49 \\
\hline $\begin{array}{c}\text { Variable Operation } \\
\text { and Maintenance } \\
\text { Costs }\end{array}$ & 0 & $\$ / \mathrm{MWh}$ & 48,49 \\
\hline
\end{tabular}


Table S35. Compressed $\mathrm{H}_{2}$ storage Assumptions for 2030 and 2050

\begin{tabular}{|c|c|c|c|}
\hline Variable & Value & Units & Source \\
\hline H2 storage CAPEX & 500 & $\$ / \mathrm{kg}$ & 50 \\
\hline $\begin{array}{c}\text { H2 storage Fixed } \\
\text { Operation and } \\
\text { Maintenance Costs }\end{array}$ & $1 \%$ & $\begin{array}{c}\% \text { of } \\
\text { CAPEX }\end{array}$ & 48,49 \\
\hline $\begin{array}{c}\text { Variable Operation } \\
\text { and Maintenance } \\
\text { Costs for charging } \\
\text { and discharging }\end{array}$ & 0.1 & $\$ /$ MWh & 48,49 \\
\hline $\begin{array}{c}\text { H2 storage }+ \\
\text { compressor } \\
\text { Lifetime }\end{array}$ & 20 & Years & 48,49 \\
\hline $\begin{array}{c}\text { H2 compressor } \\
\text { CAPEX }\end{array}$ & 1200 & \$/kWe & 51 \\
\hline $\begin{array}{c}\text { H2 compressor } \\
\text { Fixed Operation } \\
\text { and Maintenance } \\
\text { Costs }\end{array}$ & $4 \%$ & CAPEX & 50 \\
\hline
\end{tabular}

Table S36. Battery storage Assumptions for 2030

\begin{tabular}{|c|c|c|c|}
\hline Variable & Value & Units & Source \\
\hline $\begin{array}{c}\text { Battery Power } \\
\text { storage CAPEX }\end{array}$ & 810 & $\$ / \mathrm{kW}$ & 48 \\
\hline $\begin{array}{c}\text { Battery Energy } \\
\text { Storage CAPEX }\end{array}$ & 111 & $\$ / \mathrm{kWh}$ & 48 \\
\hline $\begin{array}{c}\text { Battery storage } \\
\text { Fixed Operation } \\
\text { and Maintenance } \\
\text { Costs }\end{array}$ & $2.5 \%$ & $\begin{array}{c}\% \text { of } \\
\text { CAPEX }\end{array}$ & 48 \\
\hline $\begin{array}{c}\text { Variable Operation } \\
\text { and Maintenance } \\
\text { Costs for charging } \\
\text { and discharging }\end{array}$ & 0.5 & \$MWh & 48,49 \\
\hline $\begin{array}{c}\text { Battery storage }+ \\
\text { compressor } \\
\text { Lifetime }\end{array}$ & 20 & Years & 48 \\
\hline
\end{tabular}

Table S36. Battery storage Assumptions for 2050

\begin{tabular}{|c|c|c|c|}
\hline Variable & Value & Units & Source \\
\hline $\begin{array}{c}\text { Battery Power } \\
\text { storage CAPEX }\end{array}$ & 608 & $\$ / \mathrm{kW}$ & 48 \\
\hline $\begin{array}{c}\text { Battery Energy } \\
\text { Storage CAPEX }\end{array}$ & 84 & $\$ / \mathrm{kWh}$ & 48 \\
\hline $\begin{array}{c}\text { Battery storage } \\
\text { Fixed Operation } \\
\text { and Maintenance } \\
\text { Costs }\end{array}$ & $2.5 \%$ & $\begin{array}{c}\% \text { of } \\
\text { CAPEX }\end{array}$ & 48 \\
\hline $\begin{array}{c}\text { Variable Operation } \\
\text { and Maintenance } \\
\text { Costs for charging } \\
\text { and discharging }\end{array}$ & 0.5 & $\$ / \mathrm{MWh}$ & 48,49 \\
\hline $\begin{array}{c}\text { Battery storage }+ \\
\text { compressor } \\
\text { Lifetime }\end{array}$ & 20 & Years & 48 \\
\hline
\end{tabular}




\section{References:}

(1) Keith, D. W., Holmes, G., St. Angelo, D., \& Heidel, K. (2018). A Process for Capturing CO2 from the Atmosphere. Joule. https://doi.org/10.1016/j.joule.2018.05.006

(2) Climeworks. (2017, May 31). World-first Climeworks plant: Capturing CO2 from air to boost growing vegetables [Press Release]. Retrieved from http://www.climeworks.com/wp-content/uploads/2017/05/02_PR-Climeworks-DACPlant-Case-Study.pdf

(3) Voskian, S., \& Hatton, T. A. (2019). Faradaic electro-swing reactive adsorption for CO2 capture. Energy \& Environmental Science. https://doi.org/10.1039/C9EE02412C

(4) House, K. Z., Baclig, A. C., Ranjan, M., van Nierop, E. A., Wilcox, J., \& Herzog, H. J. (2011). Economic and energetic analysis of capturing CO2 from ambient air. Proceedings of the National Academy of Sciences, 108(51), 20428-20433. https://doi.org/10.1073/pnas.1012253108

(5) Mallapragada, D. S., Gençer, E., Insinger, P., Keith, D. W., \& O’Sullivan, F. M. (2020). Can Industrial-Scale Solar Hydrogen Supplied from Commodity Technologies Be Cost Competitive by 2030? Cell Reports Physical Science, https://doi.org/10.1016/j.xcrp.2020.100174

(6) National Renewable Energy Laboratory (NREL) (2019). National Solar Radiation Database (NSRDB). https://nsrdb.nrel.gov/

(7) Brown, P. R., \& Botterud, A. (2021). The Value of Inter-Regional Coordination and Transmission in Decarbonizing the US Electricity System. Joule, 5(1), 115-134. https://doi.org/10.1016/j.joule.2020.11.013 
(8) Whitaker, M., Heath, G. A., O’Donoughue, P., \& Vorum, M. (2012). Life Cycle Greenhouse Gas Emissions of Coal-Fired Electricity Generation. Journal of Industrial Ecology, 16(s1), S53-S72. https://doi.org/10.1111/j.1530-9290.2012.00465.x

(9) O’Donoughue, P. R., Heath, G. A., Dolan, S. L., \& Vorum, M. (2014). Life Cycle Greenhouse Gas Emissions of Electricity Generated from Conventionally Produced Natural Gas. Journal of Industrial Ecology, 18(1), 125-144. https://doi.org/10.1111/jiec.12084 Moomaw, W., P. Burgherr, G. Heath, M. Lenzen, J. Nyboer, A. Verbruggen, 2011: Annex II: Methodology. In IPCC Special Report on Renewable Energy Sources and Climate Change Mitigation [O. Edenhofer, R. Pichs-Madruga, Y. Sokona, K. Seyboth, P. Matschoss, S. Kadner, T. Zwickel, P. Eickemeier, G. Hansen,S. Schlömer, C. von Stechow (eds)], Cambridge University Press, Cambridge, United Kingdom and New York, NY, USA

(11) Warner, E. S., \& Heath, G. A. (2012). Life Cycle Greenhouse Gas Emissions of Nuclear Electricity Generation. Journal of Industrial Ecology, 16(s1), S73-S92. https://doi.org/10.1111/j.1530-9290.2012.00472.x NREL (National Renewable Energy Laboratory). Life Cycle Assessment Harmonization. (n.d.). Retrieved July 26, 2020, from https://www.nrel.gov/analysis/lifecycle-assessment.html

$$
\text { Eberle, A., Heath, G. A., Carpenter Petri, A. C., \& Nicholson, S. R. (2017). }
$$

Systematic Review of Life Cycle Greenhouse Gas Emissions from Geothermal Electricity (NREL/TP--6A20-68474, 1398245; p. NREL/TP--6A20-68474, 1398245). https://doi.org/10.2172/1398245 
Greenhouse Gas Emissions of Thin-film Photovoltaic Electricity Generation. Journal of Industrial Ecology, 16(s1), S110-S121. https://doi.org/10.1111/j.1530$\underline{9290.2011 .00423 . \mathrm{x}}$ emissions from solar PV and wind energy: A critical meta-survey. Energy Policy, 65, 229-244. https://doi.org/10.1016/j.enpol.2013.10.048

$$
\text { Burkhardt, J. J., Heath, G., \& Cohen, E. (2012). Life Cycle Greenhouse Gas }
$$

Emissions of Trough and Tower Concentrating Solar Power Electricity Generation. Journal of Industrial Ecology, 16(s1), S93-S109. https://doi.org/10.1111/j.1530$\underline{9290.2012 .00474 . x}$ Utility-Scale Wind Power. Journal of Industrial Ecology, 16(s1), S136-S154. https://doi.org/10.1111/j.1530-9290.2012.00464.X United States Energy Information Administration (2019). Annual Energy Outlook 2019 with Projections to 2050 [Data file]. Retrieved from https://www.eia.gov/outlooks/archive/aeo19/

(19) Peters, M., Timmerhause, K., and West, R. (2002). Plant Design and Economics for Chemical Engineers (5 ${ }^{\text {th }}$ Edition). McGraw-Hill Education. Argonne GREET Publication: Life Cycle Analysis of Alternative Aviation Fuels in GREET. (n.d.). Retrieved July 12, 2018, from https://greet.es.anl.gov/publicationaviation-lca 
Biomass Air-Steam Gasification in a Fluidized Bed to Produce Hydrogen-Rich Gas. Energy \& Fuels, 17(3), 677-682. https://doi.org/10.1021/ef0201811

Meng, X., de Jong, W., Fu, N., \& Verkooijen, A. H. M. (2011). Biomass

gasification in a $100 \mathrm{kWth}$ steam-oxygen blown circulating fluidized bed gasifier: Effects of operational conditions on product gas distribution and tar formation. Biomass and Bioenergy, 35(7), 2910-2924. https://doi.org/10.1016/j.biombioe.2011.03.028

$$
\text { Unruh, D., Pabst, K., \& Schaub, G. (2010). Fischer-Tropsch Synfuels from }
$$

Biomass: Maximizing Carbon Efficiency and Hydrocarbon Yield. Energy \& Fuels, 24(4), 2634-2641. https://doi.org/10.1021/ef9009185

Smith R.J., Byron, Loganathan, M., \& Shantha M. S. (2010) A Review of the Water Gas Shift Reaction Kinetics. International Journal of Chemical Reactor Engineering, 8. Techno-economic analysis of biomass-to-liquids production based on gasification. (2010). Fuel, 89, S11-S19. https://doi.org/10.1016/j.fuel.2010.07.027

$$
\text { Unde, R. B. (2012). Kinetics and Reaction Engineering Aspects of Syngas }
$$

Production by the Heterogeneously Catalysed Reverse Water Gas Shift Reaction (Doctoral thesis). Retrieved from https://epub.uni-bayreuth.de/239/

$$
\text { Daza, Y. A., \& Kuhn, J. N. (2016). } \mathrm{CO}_{2} \text { conversion by reverse water gas shift }
$$
catalysis: Comparison of catalysts, mechanisms and their consequences for $\mathrm{CO}_{2}$ conversion to liquid fuels. RSC Advances, 6(55), 49675-49691. https://doi.org/10.1039/C6RA05414E

$$
\text { New York State Energy Research and Development Authority (NYSERDA). }
$$

Hydrogen Fact Sheet: Hydrogen Production - Steam Methane Reforming (SMR) [PDF]. 
Retrieved from

https://www.amiqweb.es/app/download/9343795/6hydrogenproductionsteammethanerefo $\underline{\text { rming.pdf }}$

(28) Farshchi Tabrizi, F., Mousavi, S. A. H. S., \& Atashi, H. (2015). Thermodynamic analysis of steam reforming of methane with statistical approaches. Energy Conversion and Management, 103, 1065-1077. https://doi.org/10.1016/j.enconman.2015.07.005

U.S. Department of Energy, Energy Efficiency and Renewable Energy. July 2016.

"Combined Heat and Power Technology Fact Sheet Series. Retrieved from https://www.energy.gov/sites/prod/files/2016/09/f33/CHP-Gas\%20Turbine.pdf

Ni, M., Leung, M., \& Leung, D. (2008). Technological development of hydrogen production by solid oxide electrolyzer cell (SOEC). International Journal of Hydrogen Energy, 33(9), 2337-2354. https://doi.org/10.1016/j.ijhydene.2008.02.048

Carmo, M., Fritz, D. L., Mergel, J., \& Stolten, D. (2013). A comprehensive review on PEM water electrolysis. International Journal of Hydrogen Energy, 38(12), 4901-4934. https://doi.org/10.1016/j.ijhydene.2013.01.151

IEA (2019), The Future of Hydrogen, IEA, Paris https://www.iea.org/reports/thefuture-of-hydrogen

Fu, Q., Mabilat, C., Zahid, M., Brisse, A., \& Gautier, L. (2010). Syngas production via high-temperature steam/CO2 co-electrolysis: An economic assessment. Energy \& Environmental Science, 3(10), 1382. https://doi.org/10.1039/c0ee00092b Chaumette, P., Verdon, C., and Boucot P. (1995) Influence of the hydrocarbons distribution on the heat produced during Fischer-Tropsch synthesis. Topics in Catalaysis 2, 301-311. 
of hydroprocessed renewable esters and fatty acids for jet fuel production. Biofuels, Bioproducts and Biorefining, 7(1), 89-96. https://doi.org/10.1002/bbb.1378

Bann, S. J., Malina, R., Staples, M. D., Suresh, P., Pearlson, M., Tyner, W. E., Barrett, S. (2017). The costs of production of alternative jet fuel: A harmonized stochastic assessment. Bioresource Technology, 227, 179-187. https://doi.org/10.1016/j.biortech.2016.12.032

Hombach, L. E., Doré, L., Heidgen, K., Maas, H., Wallington, T. J., \& Walther, G. (2019). Economic and environmental assessment of current (2015) and future (2030) use of E-fuels in light-duty vehicles in Germany. Journal of Cleaner Production, 207, 153-162. https://doi.org/10.1016/j.jclepro.2018.09.261 Brynolf, S., Taljegard, M., Grahn, M., \& Hansson, J. (2018). Electrofuels for the transport sector: A review of production costs. Renewable and Sustainable Energy Reviews, 81, 1887-1905. https://doi.org/10.1016/j.rser.2017.05.288 Tyner, W.E. \& Brandt, K. (2019) 2019 Techno-economic Analysis Assumptions for ASCENT Projects. FAA Center for Excellence for alternative jet fuels \& environment. US EPA, O. (2018, February 14). EGRID Summary Tables [Data and Tools]. Retrieved March 21, 2019, from US EPA website: https://www.epa.gov/energy/egrid$\underline{\text { summary-tables }}$ American Physical Society. (2011). Direct Air Capture of $\mathrm{CO}_{2}$ with Chemicals: A Technology Assessment for the APS Panel on Public Affairs. https://www.aps.org/policy/reports/assessments/upload/dac2011.pdf 
Future cost and performance of water electrolysis: An expert elicitation study. International Journal of Hydrogen Energy, 42(52), 30470-30492. https://doi.org/10.1016/j.ijhydene.2017.10.045 K. Hauptmeier, Personal Communication, June 4, 2018.

Albrecht, F. G., König, D. H., Baucks, N., \& Dietrich, R.-U. (2017). A standardized methodology for the techno-economic evaluation of alternative fuels - A case study. Fuel, 194, 511-526. https://doi.org/10.1016/j.fuel.2016.12.003

(45) Worley, M., \& Yale, J. (2012). Biomass Gasification Technology Assessment:

Consolidated Report (NREL/SR-5100-57085, 1059145). https://doi.org/10.2172/1059145

(46) Alibaba.com. Website. Retrieved November 1, 2018 from https://www.alibaba.com/showroom/calcium-carbonate-price.html

Mayyas, A. T., Ruth, M. F., Pivovar, B. S., Bender, G., \& Wipke, K. B. (2019). Manufacturing Cost Analysis for Proton Exchange Membrane Water Electrolyzers (NREL/TP-6A20-72740, 1557965; p. NREL/TP-6A20-72740, 1557965).

\section{https://doi.org/10.2172/1557965}

National Renewable Energy Laboratory (NREL) (2019). 2019 Annual

Technology Baseline. Golden, CO: National Renewable Energy Laboratory. National Renewable Energy Laboratory (NREL) (2020). 2020 Annual

Technology Baseline. Golden, CO: National Renewable Energy Laboratory. Penev, Michael and Hunter, Chad. (April 30, 2019) Energy Storage Analysis

[Presentation Slides]. NREL (National Renewable Energy Laboratory). https://www.nrel.gov/docs/fy19osti/73481.pdf 
(51) Parks, G., Boyd, R., Cornish, J., and Remick, R. (2014). Hydrogen Station Compression, Storage, and Dispensing Technical Status and Costs: Independent Review. NREL (National Renewable Energy Laboratory)

https://www.nrel.gov/docs/fy14osti/58564.pdf 